\title{
Lösungsmöglichkeiten bei fehlgeschlagenen Osteosynthesen an Schulter und Schultergürtel
}

Michael Plecko

\section{Einleitung}

Fehlschläge nach Osteosynthesen können mannigfaltige Ursachen haben und stellen meist eine große Herausforderung an das weiterbehandelnde Team dar. So können neben der Komplexität der Verletzung auch Fehleinschätzungen bei der Erstbehandlung, falsche Implantat- oder Verfahrenswahl oder mangelhafte chirurgisch-technische Ausführung, aber auch patientenspezifische Ursachen wie schlechte Knochenqualität oder mangelnde Compliance und Mitarbeit der Patientin bzw. des Patienten ursächlich für ein Fehlschlagen der Erstoperation sein. Demzufolge ist es zur erfolgreichen Behandlung unbedingt notwendig, die Ursache des Fehlschlagens gründlich zu analysieren und danach Behandlungskonzepte zu erarbeiten.

Das weitere Vorgehen ist ausführlich mit den Patienten zu diskutieren und auf die persönlichen Bedürfnisse der Person abzustimmen. Es reicht vom Akzeptieren eines verbleibenden Defizits über die Reosteosynthese mit und ohne zusätzliche unterstützende Maßnahmen (knochenheilungsfördernde Maßnahmen, Augmentation, die Stabilität fördernde Zusatzmaßnahmen) bis hin zum endoprothetischen Ersatz.

\section{Schlüsselbein}

Frakturen des Schlüsselbeines kommen mit einer jährlichen Inzidenz von ca. 30 pro 100000 Personen pro Jahr vor [1]. Die Tendenz ist im Alpenraum durch die stark zunehmende Popularität des Radfahrens und Mountainbikens deutlich steigend. Meist ist das mittlere Drittel des Schlüsselbeines betroffen (ca. 80\%), gefolgt von ca. $15 \%$ im lateralen Drittel, wobei die komplexeren Frakturformen (mehrfragmentär und/oder mit zusätzlichen Bandverletzungen) deutlich zunehmen. Dies und die steigende Evidenz, dass die konservative Therapie insbesondere bei hochenergetischen Frakturen häufig zu einer Heilung in Verkürzung des Schlüsselbeines und einer doch beträchtlichen funktionellen Einschränkung und Komplikationsrate führt [2], hat zu einer deutlichen Zunahme der operativen Versorgungen nach Schlüsselbeinfrakturen geführt. Obwohl prospektive randomisierte Vergleichsstudien ein deutlich besseres Outcome und eine geringere Komplikationsrate für die operative Versorgung im Vergleich zur konservativen Versorgung ge- zeigt haben, liegt die Komplikationsrate bei der operativen Therapie dennoch bei $37 \%$ und ist damit doch beträchtlich [3]. Die Tatsache, dass das Schlüsselbein praktisch keine intramedulläre, sondern nur eine periostale Blutversorgung aufweist [4,5] könnte neben einer zu stark traumatisierenden Operationstechnik und einer Missachtung operationstechnischer Grundregeln ein Grund von Heilungsproblemen sein. An primären Osteosynthesetechniken sind Platten und Schraubentechniken von intramedullären Verfahren zu unterscheiden, wobei allgemein die Plattenosteosynthese deutlich häufiger zur Anwendung kommt. Bei der Plattenosteosynthese kommen heute häufig vorgeformte Klavikulaplatten zum Einsatz, die meist superior oder gelegentlich auch anterior angelegt werden. Bei Beachtung einer weichteil- und periostschonenden Operationstechnik ist die Pseudarthroserate mit $1 \%$ gering [6]. Allerdings sind auch Studien mit Pseudarthroseraten bis zu 10,2\% nach Plattenosteosynthesen publiziert [7]. Sechs durch Schrauben gefasste Kortikales auf beiden Seiten der Fraktur sind empfohlen, um ein Implantatversagen zu vermeiden. Insbesondere lateral ist auf eine gute Verankerung der Schrauben zu achten, um ein Ausreißen zu vermeiden. Die Kombination von Kortikalisschrauben, die zur Seite etwas gewinkelt eingebracht werden, mit winkelstabilen Kopfverriegelungsschrauben wird von uns bevorzugt. Da die einwirkenden Kräfte am Schlüsselbein sehr groß sind, ist möglichst eine interfragmentäre Kompression (entweder durch interfragmentäre Zugschrauben oder über die Platte) und ein mechanisch robustes Implantat zu bevorzugen. Zusätzliche Risikofaktoren für eine Reoperation nach Schlüsselbeinverplattung sind Drogen- oder Alkoholmissbrauch, Diabetes mellitus und vorangegangene Schulteroperationen; ebenso wurde ein Alter über 55 als prognostisch ungünstig beschrieben [8]. Weiterhin wurde auch eine schleichende Infektion mit Cutibacterium acnes (= Propionibakterium) als Ursache bei einem hohen Prozentsatz (13 von 20 Patienten) von Schlüsselbeinpseudarthrosen gefunden [9].

Bei den intramedullären Verfahren ist die Komplikationsrate bei komplexen Frakturformen hoch und daher können diese Verfahren nur für einfache Frakturformen empfohlen werden. In der Literatur werden Pseudarthroseraten von 0 bis 9,8\% angegeben [7]. Auch Implantatbrüche kommen vor, weshalb ein Querschnitt der Pins bzw. Nägel von mindestens 2,5 mm anzustreben ist. Problema- 

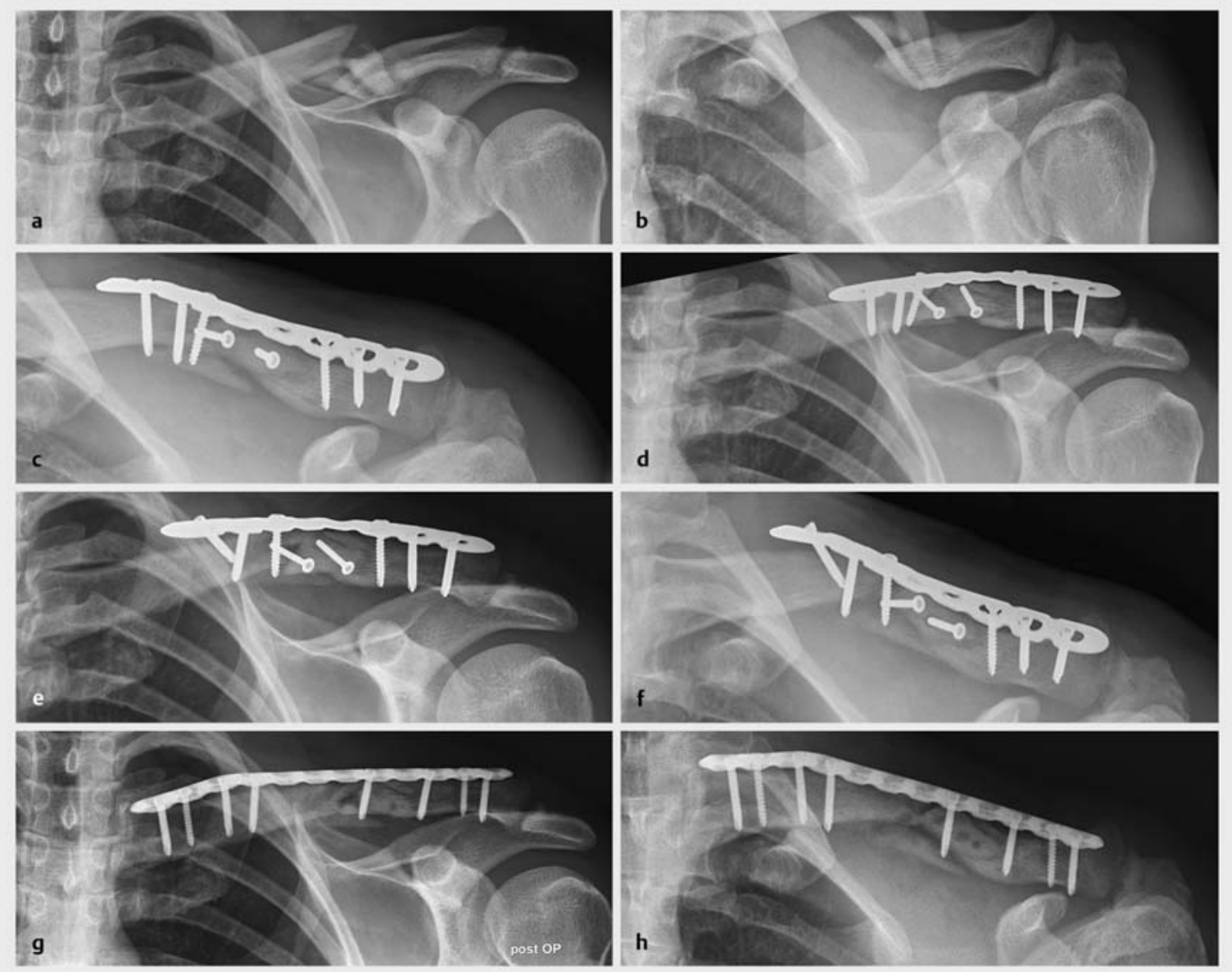

- Abb. 1 43-jähriger Patient mit einer Schlüsselbeinfraktur links. a, b Primäre Röntgenbilder. c, d Verplattung. e, f Redislokation mit Ausriss der Schrauben. $\mathbf{g}$, h Reosteosynthese mit einer längeren Platte.

tisch ist bei den nicht verriegelbaren Nägeln auch die mangelnde Rotationsstabilität, die auch zu einer Beeinträchtigung der Heilung führen kann.

Laterale Schlüsselbeinfrakturen kommen seltener vor und weisen eine Pseudarthroserate zwischen 28 und $44 \%$ auf, insbesondere nach konservativer Behandlung [10], wobei nur ein Teil davon symptomatisch ist. Die Ursache bei dislozierten Frakturen ist meist die hohe Instabilität der Frakturen, bei denen auch der Bandapparat, insbesondere die korakoklavikulären (CC) Bänder mitverletzt sind. In solchen Fällen führt die Plattenosteosynthese kombiniert mit einer Sicherung der korakoklavikulären Bänder durch eine CC-Schraube oder eine zusätzliche CCBandschlinge zu einer guten Heilung. Hierbei wurde gezeigt, dass die Hakenplatte und die winkelstabile distale Klavikulaplatte zu gleich guten klinischen Ergebnissen führen, mit einer etwas höheren Komplikationsrate bei Anwendung der Hakenplatte [11]. Eine frühzeitige Versorgung ist anzustreben, da eine verzögerte Versorgung zu höheren Komplikationsraten führt $(7,4 \%$ in der akut versorgten Gruppe vs. 36,4\% in der verspätet versorgten Gruppe) [12]. Die häufigsten Komplikationen waren periimplantäre Frakturen, Infektionen und ein Implantatversagen. In einer geringen Anzahl der operierten Fälle (2 von 38) kam es zu einer Pseudarthrose.

\section{Behandlung}

Patienten mit Implantatversagen, Implantatbruch oder Ausriss bzw. Auslockern der Schrauben nach Plattenosteosynthese werden, so es die Knochenqualität erlaubt, mit einer Reosteosynthese mit einer neuen, längeren Platte versorgt ( $\triangleright$ Abb. 1). Wir bevorzugen hier die Kombination von Kortikalisschrauben, die divergent eingebracht werden, mit winkelstabilen Kopfverriegelungsschrauben. Gelegentlich ist ein Wechsel der Plattenposition notwendig. Eine Infektion muss immer durch Entnahme von mehreren Gewebeproben ausgeschlossen werden. Wenn eine lokale Durchblutungsproblematik vorliegt, wird der Knochen zusätzlich angefrischt und eine autologe Spongiosa aus dem Beckenkamm beigelegt ( Abb. 2). Wachstumsfaktoren oder Stammzellen 


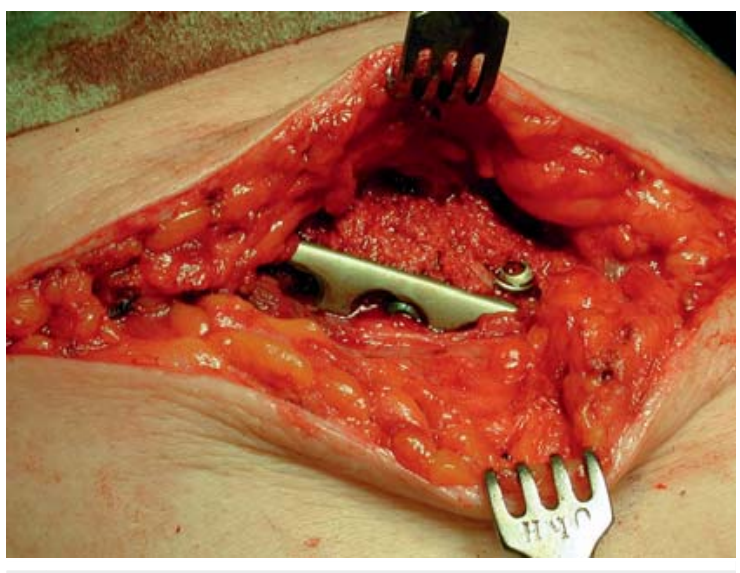

- Abb. 2 Plattenosteosynthese mit Spongiosaplastik.

werden bei uns im Routinebetrieb lokal am Schlüsselbein nicht angewandt. Wichtig ist es, eine ausreichende Stabilität zu erzielen. Problematisch sind oft bei der Reoperation die Nn. supraclaviculares, die manchmal im Narbengewebe nur schwer identifiziert und geschont werden können.

Bei peripheren Schlüsselbeinfrakturen, bei denen eine Plattenosteosynthese versagt, kann bei ausreichend vorhandenem Knochen eine Reosteosynthese entweder mit einer Hakenplatte, einer winkelstabilen distalen Klavikulaplatte in Kombination mit einer vom Schlüsselbein zum Korakoid geführten Bandaugmentation oder durch eine Doppelplattenosteosynthese (superior und anterior) durchgeführt werden. Bei Knochendefekten nach Trümmerzonen kann eine Spongiosaplastik beigefügt werden [13] ( Abb. 3). Bei unzureichender Knochenqualität und weit lateral gelegenen Frakturen kann auf eine modifizierte Weaver-Dunn-Operation mit Resektion des peripheren Fragmentes und Stabilisierung des Schlüsselbeines mit dem eingeschwenkten Lig. coracoacromiale zurückgegriffen werden. Diese Technik hat in der Behandlung der Akromioklavikulargelenkverrenkung gute Ergebnisse gezeigt [14].

Beim Versagen einer Osteosynthese nach intramedullärer Versorgung mit ausgelockertem Implantat ist ein Verfahrenswechsel mit Umstieg auf eine Plattenosteosynthese zu bevorzugen. Es sollte versucht werden, interfragmentäre Kompression zu erzeugen, und bei schlechter lokaler Biologie wird der Knochen angefrischt und autologe Spongiosa beigelegt. Bei abgebrochenem intramedullären Pin (ESIN - elastisch-stabiler intramedullärer Nagel) kann es schwierig sein, den peripheren Anteil aus dem Markraum zu entfernen, insbesondere wenn Titannägel länger in situ waren und eingewachsen sind. Ein Versuch, diesen Anteil vom nicht geheilten Frakturspalt aus zu entfernen, ist gerechtfertigt. Ansonsten kann versucht werden, bei liegenden Implantatrest dennoch eine Platten- osteosynthese durchzuführen und die Schrauben am Draht vorbei zu platzieren.

Bei Pseudarthrosen mit Knochendefekt ist unabhängig von der Erstbehandlung (Platte, intramedulläre Stabilisierung oder konservative Behandlung) ein Eröffnen des Markraumes und Anfrischung des Knochens bzw. eine Resektion der abgedeckelten Knochenenden durchzuführen. Der entstandene Knochendefekt ist dann mit einem kortikospongiösen Knochenblock, bevorzugt aus dem Beckenkamm, zu überbrücken $[15,16]$. Auch freie gefäßgestielte Knochentransplantate aus der Femurkondyle oder alternativ eine freie Fibula sind beschrieben $[17,18]$ ( $>$ Abb.4). Neben der stabilen Plattenosteosynthese ist eine interfragmentäre Kompression an den Kontaktflächen vorteilhaft, weshalb wir trapezartig geformte Knochenblöcke bevorzugen. Die ursprüngliche Länge und Form des Schlüsselbeines muss möglichst wiederhergestellt werden [19]. Daher ist eine genaue präoperative Planung unumgänglich, wobei meist die gesunde Gegenseite als Template herangezogen wird. Die Planung erfolgt heute meist CT-basiert bzw. in spezialisierten Zentren computerunterstützt $(\triangleright \mathbf{A b b}$. 5). Bei schlechter Weichteildeckung muss ein plastisch-rekonstruktiver Eingriff erwogen werden. Ein lateraler Teil des klavikulären Anteils des M. pectoralis kann über den Pseudarthrosebereich eingeschwenkt werden.

\section{Ergebnisse}

Bei korrekter Analyse der Gründe des Fehlschlagens der Erstbehandlung und unter Beachtung der technischen Details kann nach Reosteosynthese am Schlüsselbein mit und ohne Knochenbeilage dennoch mit einem guten Ergebnis gerechnet werden. Die verlängerten Heilungszeiten nach Knochenaufbau bzw. auch nach Infektionen sind zu beachten. Die Angaben in der Literatur zu diesem Thema sind sehr spärlich $[13,16]$, sodass auf keine ausreichende Evidenz zurückgegriffen werden kann; allerdings ist im Vergleich zur operativen Versorgung von frischen Frakturen bei Pseudarthrosen mit einem mehr als doppelt so hohen Risiko einer frühen postoperativen Komplikation zu rechnen [20].

\footnotetext{
Merke

Ein Versagen einer operativen Versorgung von Schlüsselbeinfrakturen ist selten und meist auf eine fehlerhafte Einschätzung der Verletzung, eine mangelhafte Operationstechnik und/oder patientenspezifische Faktoren wie mangelnde Compliance oder schlechte Knochenqualität sowie eine schleichende Infektion zurückzuführen. Bei exakter Analyse der Ursache und sachgemäßer operativer Sanierung i. d. R. durch Reosteosynthese und autologe Knochentransplantation ist mit einer guten Heilung und einem guten funktionellen Ergebnis zu rechnen.
} 

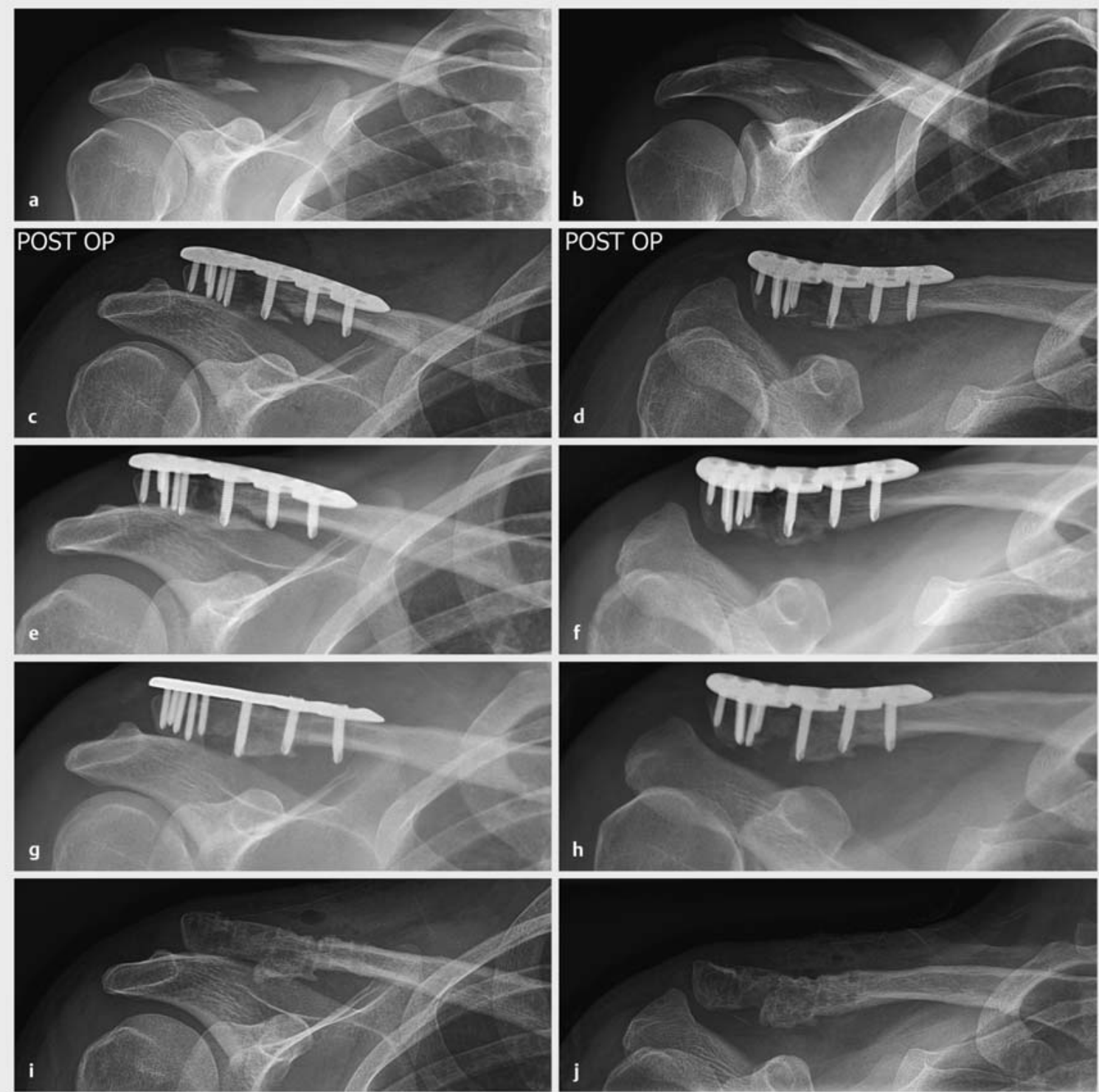

Abb. 3 Periphere Klavikulafraktur. a, b Primäre Röntgenbilder. c, d Verplattung der Fraktur mit einer winkelstabilen Platte. e, f Pseudarthrose mit Knochenresorption. g, h Reoperation mit interponiertem Spongiosablock. i,j Röntgenbilder nach Implantatentfernung.

\section{Schulterblatt}

Schulterblattfrakturen sind meist das Resultat von hochenergetischen Traumen und betreffen ca. $1 \%$ aller Frakturen und ca. 3\% aller Verletzungen des Schultergürtels. Circa $70 \%$ betreffen den Schulterblattkörper, Schulterblatthals und die Spina und ca. 10\% die Fossa glenoidalis [21]. Sie können in einem hohen Prozentsatz konservativ behandelt werden. Eine relevante mediolaterale Verschiebung zwischen Glenoid und dem Margo lateralis der Skapula von mehr als $2 \mathrm{~cm}$, eine Abknickung der Skapula in der Outlet-Projektion von mehr als $45^{\circ}$, ein glenopolarer Winkel von $20^{\circ}$ oder weniger sowie Stufenbildun- gen an der glenoidalen Gelenkfläche werden heute als Operationsindikationen angesehen. Am Glenoid steht die Verschraubung im Vordergrund, während bei kleineren ventralen Pfannenabbrüchen auch mehr und mehr arthroskopische Techniken zur Anwendung kommen. Bei dislozierten Frakturen des Schulterblattkörpers wird eine offene Reposition über limitierte Zugänge von dorsal favorisiert und eine Abstützung mit Platten zwischen Skapulahals und Margo lateralis, an der Spina selbst sowie am Übergang der Spina zum Margo medialis angestrebt. Ein Versagen der Osteosynthese ist weder im eigenen Patientenkollektiv beobachtet worden, noch in der Literatur in relevanter Anzahl beschrieben. Sollte im Einzelfall eine 


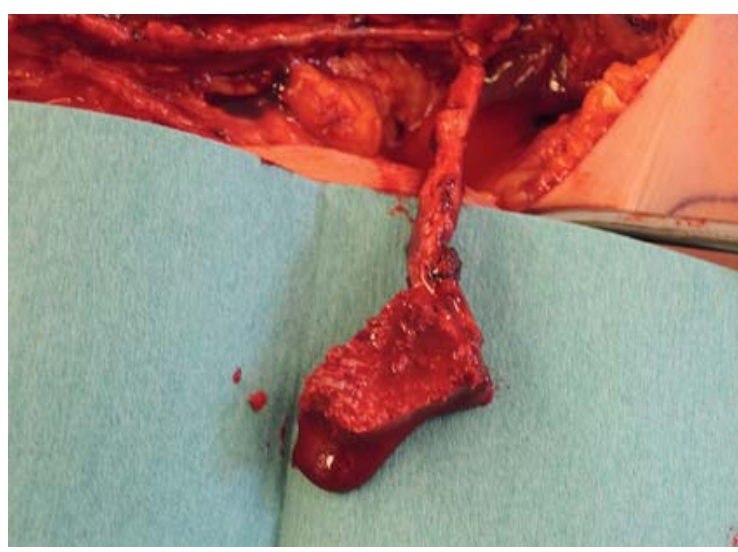

- Abb. 4 Gefäßgestielter Knochenspan aus der Femurkondyle.

relevante Redislokation eintreten, kann diese nur durch neuerliche Reposition und Reosteosynthese adressiert werden. Häufig führt ein Implantatversagen, z. B. Plattenbruch, dennoch zur knöchernen Heilung und nur gelegentlich ist die Implantatentfernung angezeigt [22]. Somit scheint die Osteosynthese der Skapulafrakturen bei korrekter Indikationsstellung und exakter technischer Durchführung in der überwiegenden Mehrzahl der Fälle zu einer guten Heilung und zu guten funktionellen Ergebnissen zu führen [23-27]. Reosteosynthesen sind kaum nötig. Gelegentlich kann im Langzeitverlauf nach intraartikulären Frakturen durch fortschreitende Arthrose die Implantation einer Schultergelenktotalendoprothese notwendig sein.

\section{Merke}

Osteosynthesen im Bereich des Schulterblattes führen selten zum Implantatversagen oder zu Fehlschlägen, die eine Revisionsoperation erfordern. Bei guter Reposition und Schrauben- sowie Plattenosteosynthese heilen diese i.d. R. mit guten funktionellen Ergebnissen. Selten kann im Langzeitverlauf bei progredienter Arthrose nach intraartikulären Frakturen eine Schultergelenkendoprothese sinnvoll sein.

\section{Proximaler Humerus}

Frakturen am proximalen Humerus, insbesondere am Humeruskopf, sind mit ca. 5\% aller Frakturen häufige Verletzungen und ihre Häufigkeit nimmt aufgrund der Verschiebung der Alterspyramide stetig zu. Sie gelten als sog. Indikatorfrakturen für Osteoporose und betreffen häufiger Frauen im fortgeschrittenen Lebensalter als Männer. Ihre Inzidenz wird in europäischen Ländern mit 392 Frakturen auf 100000 Einwohner/Jahr für Frauen älter als 65 und mit 520 Frakturen auf 100000 Einwohner/ Jahr für Frauen älter als 80 angegeben [28]. Bezüglich der Behandlung besteht noch immer beträchtliche Uneinig- keit. Neben der konservativen Therapie kommen perkutane, minimalinvasive operative Verfahren, die Marknagelung, die offene Rekonstruktion und Plattenosteosynthese sowie die Endoprothetik als Frakturprothese oder inverse Schulterendoprothese infrage [29-37].

Das Ziel jeder Osteosynthese am proximalen Humerus ist die möglichst anatomische Reposition, ohne zusätzliche Schädigung der Fragmentdurchblutung und die Stabilisierung bis zur knöchernen Heilung. Die verschiedenen zur Verfügung stehenden Verfahren haben jeweils unterschiedliche Vor- und Nachteile und wenngleich mit allen Verfahren über gute Ergebnissen berichtet wird, so gibt es doch Unterschiede in der Art und Häufigkeit von Komplikationen. Diese sind außerdem zusätzlich von der Frakturform und der Knochenqualität abhängig. Eine Indikation zur Revisionsoperation ist generell abhängig von Art und Ausmaß einer Redislokation, von der Knochenqualität und etwaigen Defekten, dem Weichteilstatus sowie dem funktionellen Anspruch des Patienten, seinem Gesundheitsstatus und seinen Komorbiditäten sowie von der Schmerzintensität.

Die perkutanen Verfahren, insbesondere die gedeckte Reposition und die Stabilisierung mit perkutan eingebrachten Pins und kanülierten Schrauben, stellen hohe Anforderungen an das Verständnis der Fraktur. Daher ist es oft technisch schwierig, eine ausreichend gute Reposition zu erzielen und die wichtigen Fragmente auch ausreichend stabil zu fassen. Das Auswandern der Bohrdrähte kann durch zusätzliche Implantate wie den Humerusblock verhindert werden. Dies führt aber bei vielen Fällen durch das Sintern der Fraktur zur sekundären Perforation der Drahtspitzen in das Gelenk. In einem solchen Fall ist das Zurückziehen der Drähte ausreichend, um die Situation zu lösen. Schwieriger ist es, wenn es zur Redislokation oder zum Abkippen der Fragmente kommt oder bei der Erstoperation keine ausreichende Reposition erzielt werden konnte. Hier ist in unseren Händen oft die offene Revisionsoperation, Reposition und Reosteosynthese die einzige Möglichkeit, noch ein gutes Ergebnis zu erzielen ( $\triangleright$ Abb. 6). Natürlich spielt die Art und das Ausmaß der Redislokation eine Rolle für die Indikation zu einem weiteren Eingriff. Eine Stufenbildung an der Gelenkfläche ist in jedem Fall zu vermeiden und über $2 \mathrm{~mm}$ inakzeptabel. Hier streben wir eine offene anatomische Reposition an, wobei oft das Eröffnen des Rotatorenintervalls zur digitalen Kontrolle der Gelenkfläche notwendig ist.

Ist es zu einer Redislokation des Tuberculum majus gekommen, so hängt das Vorgehen vom Ausmaß der Verschiebung und vom Alter des Patienten ab. Bei aktiven Patienten stellt für uns das Höherwandern des Tuberculum majus von 5 mm oder mehr eine Indikation zur Reoperation dar. Die gelockerten Schrauben lassen sich i.d.R. leicht ohne weiteren Schaden entfernen, wir passen dann das Fragment an anatomischer Stelle wieder 

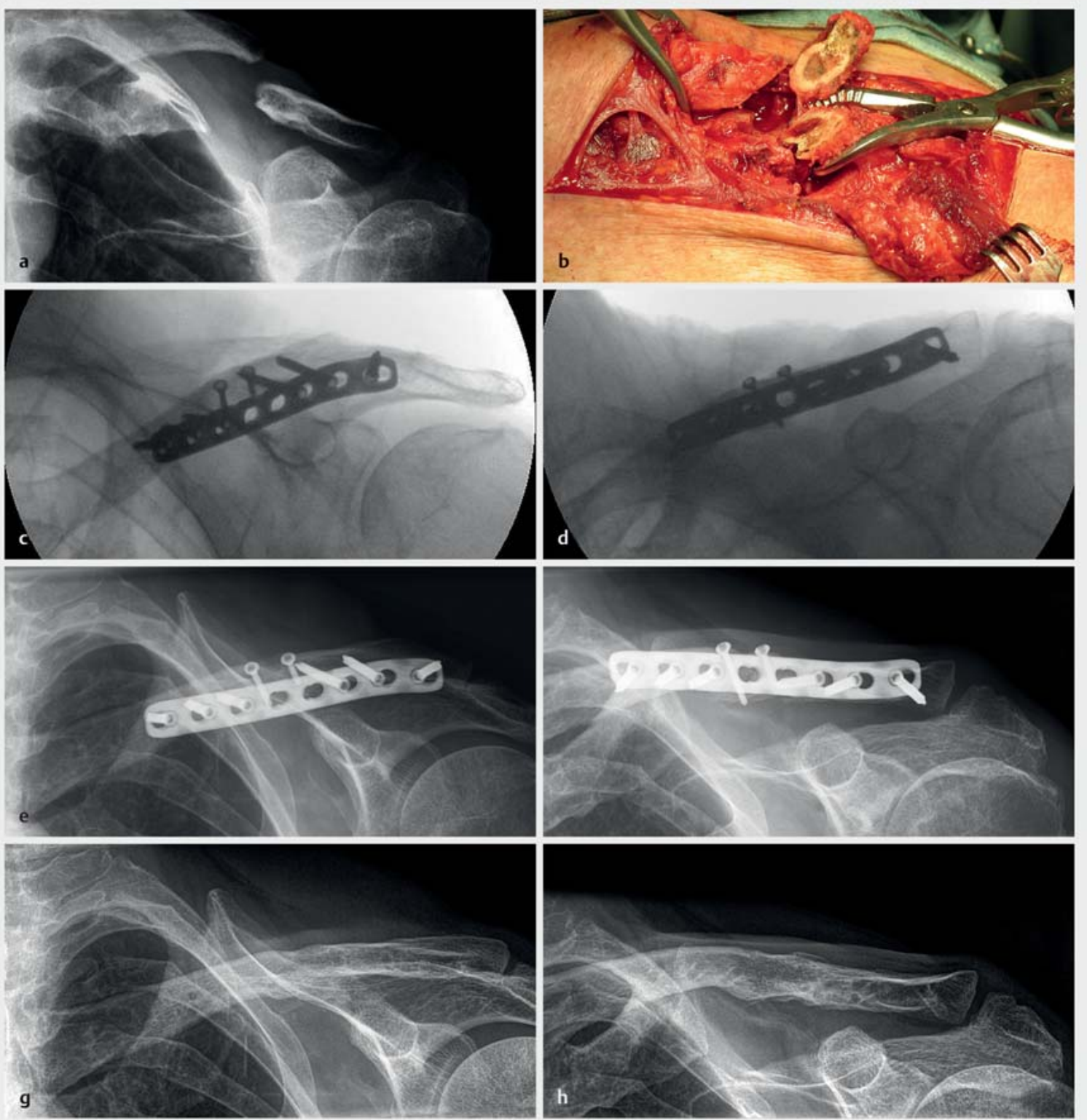

- Abb. 5 73-jähriger Patient mit Pseudarthrose nach Erstversorgung. a Röntgenbild. b Intraoperativer Situs - Resektion der Pseudarthrose und Eröffnen des Markkanals. c, $\mathbf{d}$ Intraoperative Bildwandlerbilder. e, $\mathbf{f}$ Verplattung mit interponiertem kortikospongiösen Knochenblock. g, h Röntgenbild nach Implantatentfernung.

ein und fixieren es je nach Größe mit einer winkelstabilen Platte und zusätzlichen Zuggurtungsschlingen oder kanülierten Schrauben mit zusätzlicher Zuggurtung. Bei kleinen Fragmenten, die wie ein knöcherner Rotatorenmanschettenausriss zu werten sind, bevorzugen wir eine 2-reihige Nahttechnik mit transossären Nähten und einer medialen Ankerreihe und Verspannung der Fäden im Sinne einer Bridge-Technik. Wir streben eine Korrekturoperation bei gegebener Indikation so rasch wie möglich an, da bei chronischer Dislokation des Tuberculums durch die
Weichteilkontraktur eine Reposition schwierig bis unmöglich wird.

Ein Absinken des Kopffragmentes in Varusposition ist beim aktiven Patienten nur in geringem Ausmaß tolerabel. Eine Varusfehlstellung von mehr als $45^{\circ}$ und ein entsprechender Funktionsanspruch des Patienten stellt für uns eine Indikation zur Korrektur dar. Die Varusstellung führt zu Schmerzen und Bewegungseinschränkung sowie zu einem Impingement. Bei veralteten Fällen kommt eine 
Akromioplastik mit zusätzlicher Tuberculoplastik infrage; bei ausgeprägten Fällen die Korrekturosteotomie und Plattenosteosynthese. Bei rezenten Fällen und vitalem Kopffragment wird eine offene Reposition und Stabilisierung mit einer winkelstabilen Plattenosteosynthese angestrebt. Bei Defekten im Kalkarbereich stellen wir durch ein zusätzlich zur Platte eingebrachtes Knochentransplantat (bevorzugt Fibula-Allograft) die mediale Abstützung wieder her. Beim betagten Patienten und fraglich vitalem oder avitalem Kopffragment bevorzugen wir in der Revisionssituation die inverse Schulterprothese, wenngleich die Ergebnisse der Endoprothetik nach fehlgeschlagener Osteosynthese schlechter vorhersehbar sind als die der primären Frakturendoprothese [38, 39].

Intramedulläre Stabilisierungen proximaler Humerusfrakturen mit speziellen proximalen Humerusnägeln können insbesondere bei metaphysären Trümmerzonen und intaktem Kopffragment (A3-Frakturen) gute Ergebnisse erzielen. Sekundäre Dislokationen und Durchschneiden der Verriegelungsschrauben durch das Kopffragment treten bei osteoporotischem Knochen auf. Auch kann es in solchen Fällen zu einem langsamen Höherwandern des $\mathrm{Na}$ gelendes und einem sekundären Implantatimpingement kommen. In Abhängigkeit vom Aktivitätsgrad des Patienten und seinem Gesundheitszustand sollte frühzeitig ein Implantatwechsel oder die Entscheidung zum Umstieg auf eine inverse Schulterprothese getroffen werden. Wenn Verriegelungsschrauben durch die Kopfkalotte perforieren, sollte rasch revidiert werden, da es sehr schnell zu einem beträchtlichen Pfannendefekt kommen kann, der dann die prothetische Versorgung sehr erschwert. Bei Redislokation, z. B. durch Lockern des Nagels und der Bolzen, kann bei ausreichender Knochenqualität auch eine Reosteosynthese mit einer winkelstabilen Platte, in manchen Fällen mit einem zusätzlichen Knochengraft, durchgeführt werden ( $\bullet$ Abb. 7).

Auch bei der winkelstabilen Plattenosteosynthese sind in der Literatur hohe Komplikationsraten beschrieben [40]. Viele dieser Komplikationen sind auf mangelnde Operationstechnik oder insuffiziente Reposition zurückzuführen. Sekundär dislozierte Tubercula, insbesondere Tuberculum-majus-Fragmente, müssen frühzeitig revidiert und refixiert werden, um ein schlechtes funktionelles Ergebnis abzuwenden. Bei länger verzögerter Reoperation ist es durch die rasche Retraktion der Muskulatur und die rasche Kontraktur dann oft nicht mehr möglich, das Fragment an der ursprünglichen Stelle zu fixieren und hier zur Heilung zu bringen. Zur Fixierung verwenden wir i. d. R. kräftige Tapes, die am Sehnen-Knochen-Übergang in einer hinterstochenen Nahtweise fixiert werden. Lateral werden diese an der Platte fixiert. Bei größeren Fragmenten kann zusätzlich eine Schraubenosteosynthese erfolgen.

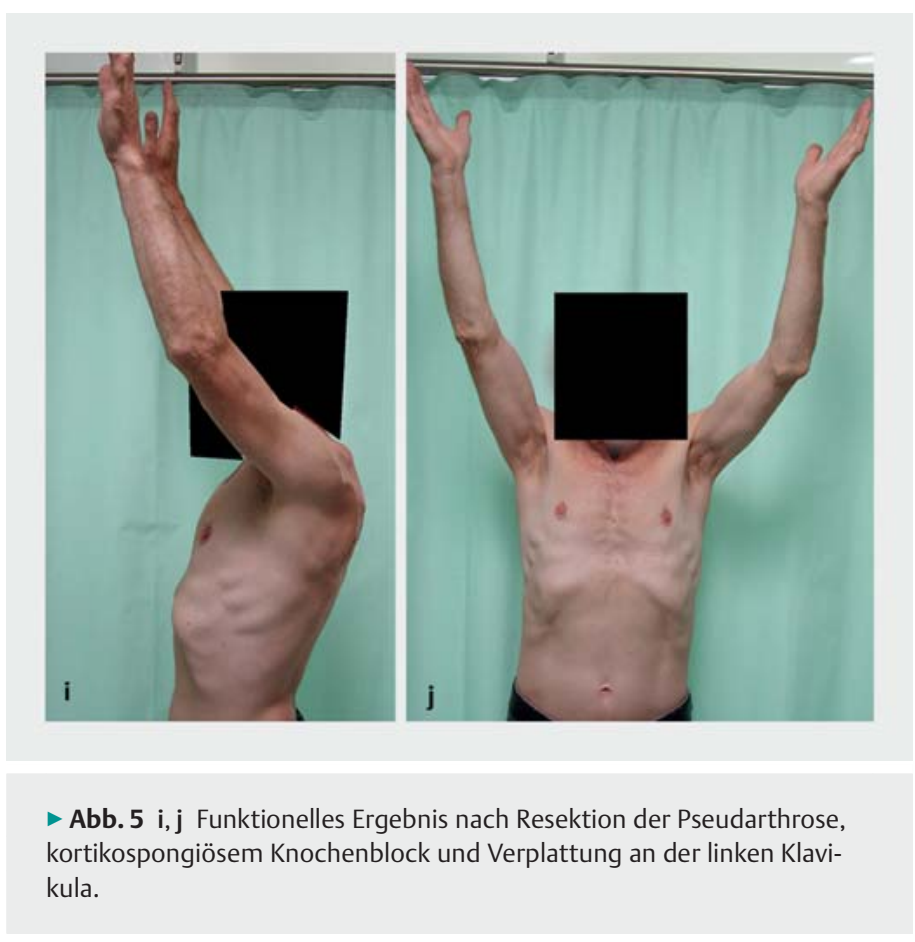

Eine Missachtung der medialen Abstützung führt am proximalen Humerus häufig bei zusätzlich schlechter Knochenqualität zu einer frühen Redislokation und sekundären Schraubenperforation ins Gelenk ( $\bullet$ Abb. 8). Bei perforierten Schraubenspitzen ist eine frühe Revisonsoperation angezeigt, da auch hier sehr rasch ein beträchtlicher Schaden am Glenoid mit erheblichen Pfannendefekten resultiert. Bei weitgehend korrekter Position der Fragmente kann dies eine reine Schraubenentfernung oder ein Schraubenwechsel über Stichinzisionen sein. Bei Dislokation des Kopffragmentes und fehlender medialer Abstützung ist, je nach Durchblutung und Knochenbeschaffenheit des Kopffragmentes und je nach Funktionsanspruch des Patienten, die Entscheidung zur Reosteosynthese mit Fibulagraft [41] oder zum Umstieg auf eine Prothese zu treffen.

Auch hier sind die funktionellen Ergebnisse der Endoprothese weniger vorhersehbar und im Durchschnitt etwas schlechter als nach der primären Frakturendoprothetik, wenngleich gute Schmerzreduktion erreicht werden kann, weshalb bei geriatrischen Patienten die primäre Entscheidung zwischen konservativer, operativ rekonstruktiver und primär endoprothetischer Versorgung sehr bewusst getroffen werden muss $[42,43]$. Die inverse Schulterendoprothetik stellt jedoch insbesondere bei älteren Patienten eine sehr gute Revisionsoption dar, um nach fehlgeschlagenen Osteosynthesen ein noch gutes Ergebnis für den Patienten erzielen zu können, wenngleich ein etwas höheres Komplikationsrisiko besteht [44]. 

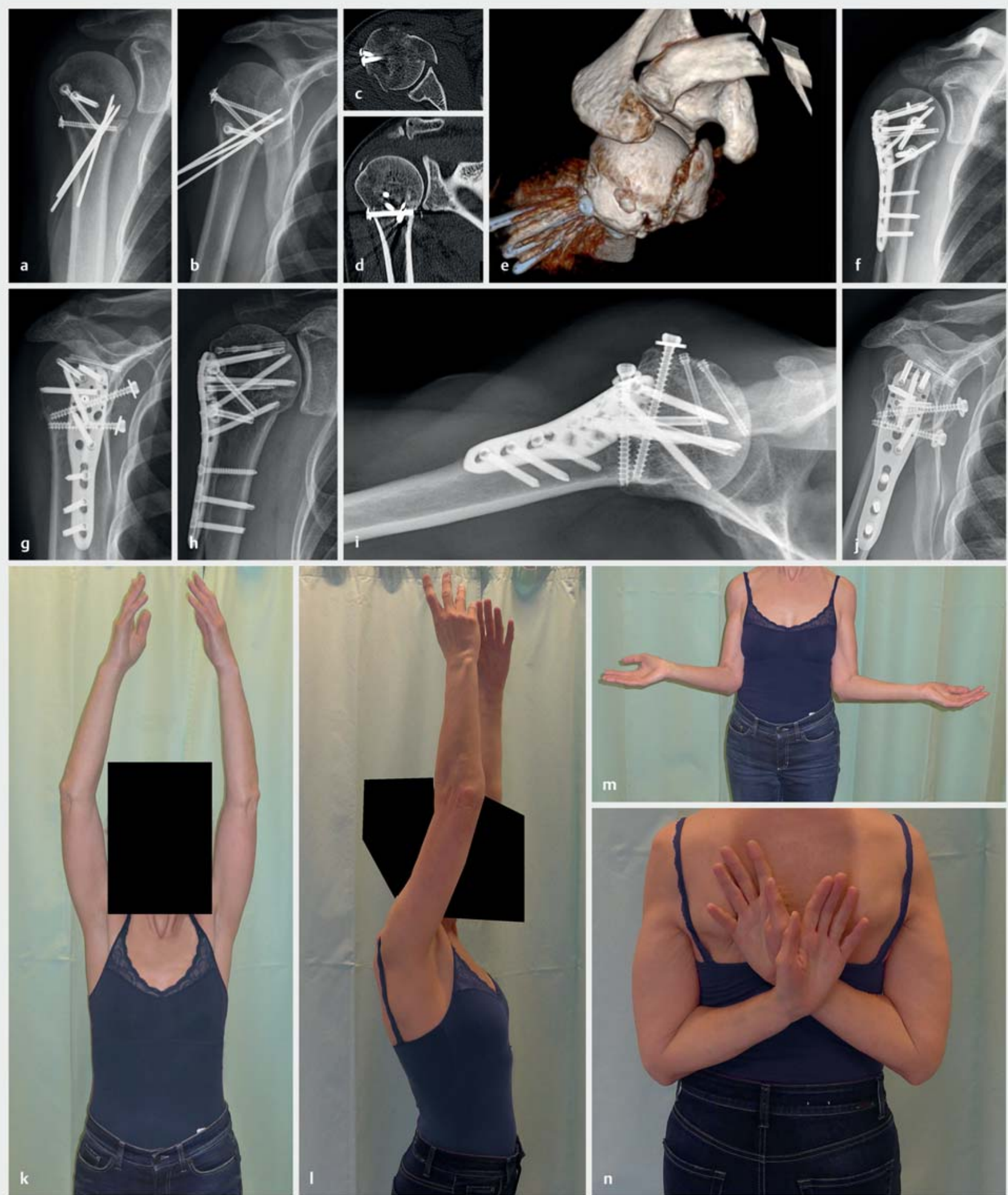

- Abb. 6 61-jährige sportliche Patientin nach Skisturz - perkutane Versorgung einer proximalen Humerusfraktur auswärts. a, b Röntgen nach Erstversorgung. c-e CT nach Erstversorgung. f, g Röntgen postoperativ nach Reosteosynthese offen. $\mathbf{h - j}$ Röntgen nach 2 Jahren. k-n Funktionelles Ergebnis. 

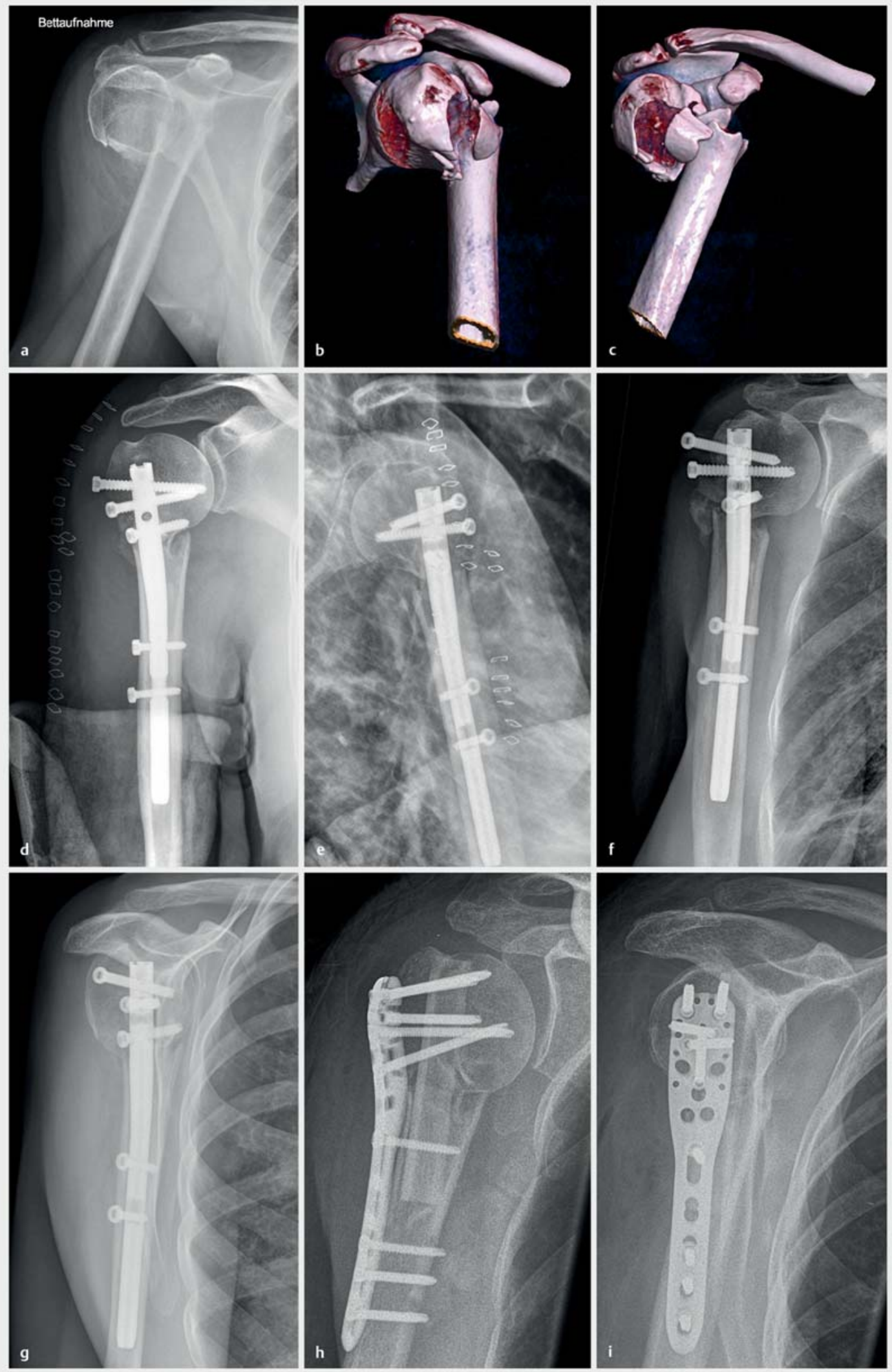

- Abb. 7 67-jährige Patientin mit proximaler Humerusfraktur. a Primäres Röntgenbild. b, c Primäres CT. d, e Stabilisierung mit einem Verriegelungsmarknagel (PHN). f, $\mathbf{g}$ Redislokation der Fraktur bei Implantatlockerung. h, i Reoperation mit Fibulagraft und winkelstabiler Humerusplatte. 

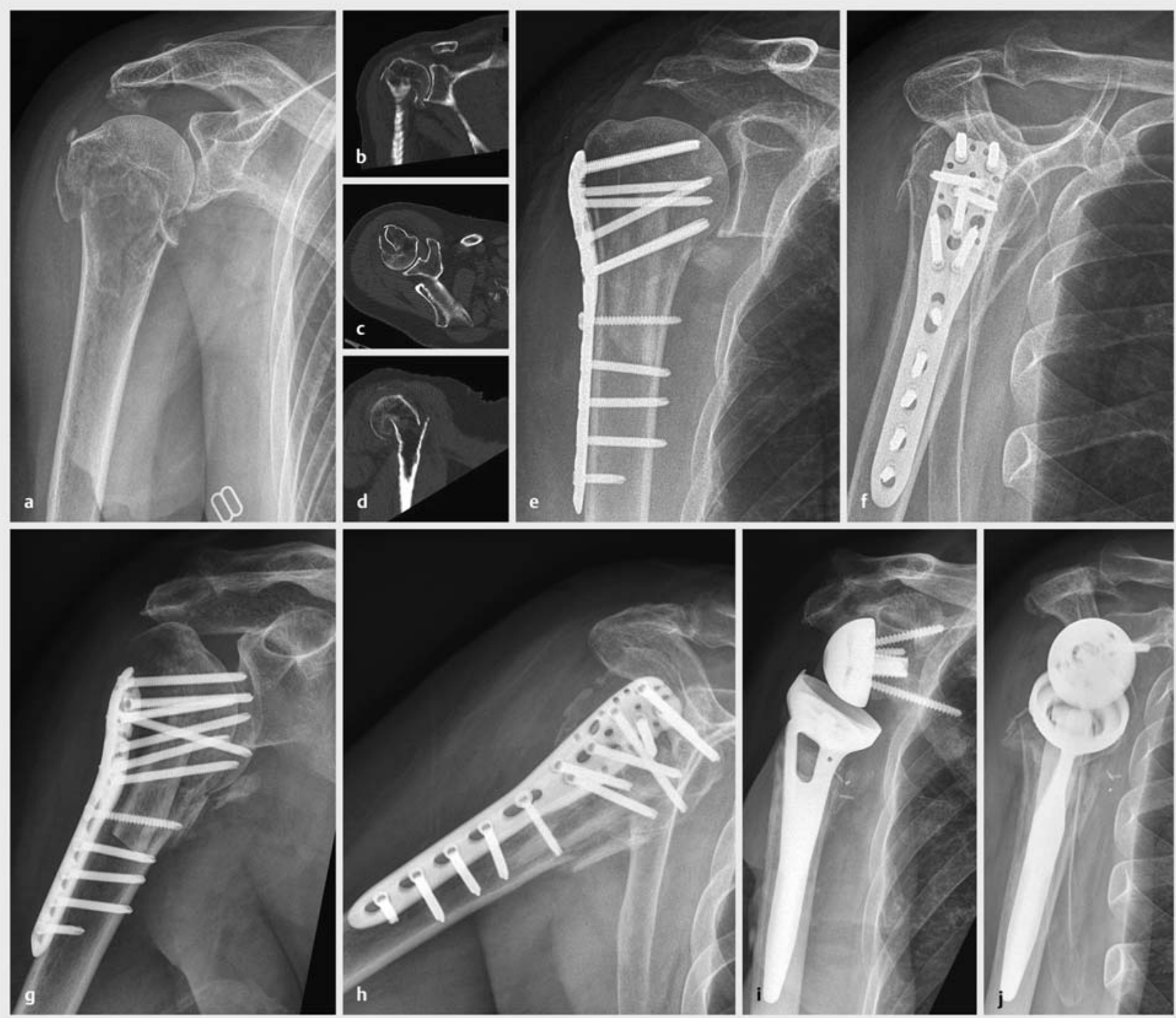

- Abb. 8 70-jährige Patientin nach häuslichem Sturz. a Primäres Röntgenbild. b-d Primäres CT. e, f Stabilisierung mit PHILOS-Platte und intramedullärem Fibulaspan. $\mathbf{g}$, h Redislokation und Perforation der winkelstabilen Schrauben. $\mathbf{i}, \mathbf{j}$ Implantation einer inversen Schultergelenkendoprothese.

\section{Ergebnisse}

Frühzeitig revidierte sekundäre Redislokationen führen bei geeigneter Technik i.d.R. zu guten Ergebnissen und guten Heilungsraten. Im eigenen Patientenkollektiv inverser Frakturendoprothesen konnte kein signifikanter Unterschied zwischen den primär und den sekundär implantierten Prothesen gefunden werden. Lediglich die Beweglichkeit war, obwohl zufriedenstellend, bei den sekundären Prothesen minimal geringer. Auch die Komplikationsrate war in beiden Gruppen mit 2 bzw. 3\% nicht signifikant unterschiedlich. Mit der inversen Schulterendoprothese konnte im Revisionsfall nach fehlgeschlagener Osteosynthese in der überwiegenden Mehrzahl der Fälle ein sehr zufriedenstellendes Ergebnis erzielt werden.

\section{Merke}

Bei fehlgeschlagenen Osteosynthesen am proximalen Humerus kommt es auf den Grad der Dislokation, das betroffene Fragment (Tuberculum majus, minus oder Kopffragment) und die Knochenqualität bzw. die Durchblutung an, ob ein kopferhaltendes Verfahren sinnvoll ist oder der Umstieg auf eine Endoprothese (bevorzugt heute eine inverse Schulterendoprothese) gewählt wird. 


\section{Schlussfolgerung}

Fehlschläge nach Osteosynthesen an Schultergelenk und Schultergürtel bedürfen einer exakten Analyse und eines patientenspezifischen Vorgehens, das immer die funktionellen Bedürfnisse des Patienten im Auge haben muss. Es sind stets Einzelfallentscheidungen, ob nochmals eine Reosteosynthese mit oder ohne Augmentation und Spongiosaplastik sinnvoll erscheint oder ein befriedigendes Therapieziel besser durch den Umstieg auf eine Schulterendoprothese zu erzielen ist. Bei geeigneter Therapiewahl kann i.d.R. ein für den Patienten befriedigendes Endergebnis erzielt werden.

\section{Interessenkonflikt}

Die Autorinnen/Autoren geben an, dass kein Interessenkonflikt besteht.

\section{Autorinnen/Autoren}

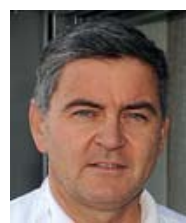

\section{Prim. Dr. med. Michael Plecko}

Ärztlicher Direktor des Unfallkrankenhauses Steiermark der AUVA, Abteilungsvorstand Abteilung Orthopädie und Traumatologie Standort Graz, Lehrkrankenhaus der MEDUNI Graz

\section{Korrespondenzadresse}

Prim. Dr. med. Michael Plecko

Ärztlicher Direktor

Unfallkrankenhaus Steiermark der AUVA

Lehrkrankenhaus der MEDUNI Graz

Göstingerstraße 24

8020 Graz

Österreich

michael.plecko@auva.at

\section{Literatur}

[1] Robinson CM. Fractures of the clavicle in the adult: Epidemiology and classification. J Bone Joint Surg Br 1998; 80: 476-484

[2] Hill JM, McGuire MH, Crosby LA. Closed treatment of displaced middle-third fractures of the clavicle gives poor results. J Bone Joint Surg Br 1997; 79: 537-539

[3] Canadian Orthopaedic Trauma Society. Nonoperative treatment compared with plate fixation of displaced midshaft clavicular fractures. A multicenter, randomized clinical trial. J Bone Joint Surg Am 2007; 80: 1-10

[4] Knudsen FW, Andersen M, Krag C. The arterial supply of the clavicle. Surg Radiol Anat 1989; 11: 211-214

[5] Havet E, Duparc F, Tobenas-Dujardin AC et al. Vascular anatomical basis of clavicular non-union. Surg Radiol Anat 2008; 30: $23-28$

[6] McKee RC, Whelan DB, Schemitsch EH et al. Operative versus nonoperative care of displaced midshaft clavicular fractures: a meta-analysis of randomized clinical trials. J Bone Joint Surg Am 2012; 94: 675-684
[7] Liu HH, Chang CH, Chia WT et al. Comparison of plates versus intramedullary nails of displaced midshaft clavicular fractures. J Trauma 2010; 69: E82-E87

[8] Schemitsch LA, Schemitsch EH, Kuzyk P et al. Prognostic factors for reoperation after plate fixation of the midshaft clavicle. J Orthop Trauma 2015; 29: 533-537

[9] Gausden EB, Villa J, Warner S] et al. Nonunion after clavicle osteosynthesis: high incidence of Propionibacterium acnes. J Orthop Trauma 2017; 31: 229-235

[10] Banerjee R, Waterman B, Padalecki J et al. Management of distal clavicle fractures. J Am Acad Orthop Surg 2011; 19: 392401

[11] Zhang C, Huang J, Luo Y et al. Comparison of the efficacy of a distal clavicular locking plate versus a clavicular hook plate in the treatment of unstable distal clavicle fractures and a systematic literature review. Int Orthop 2014; 38: 1461-1468

[12] Klein SM, Badman BL, Keating C] et al. Results of surgical treatment for unstable distal clavicular fractures. J Shoulder Elbow Surg 2010; 19: 1049-1055

[13] Villa JC, van der List JP, Gausden EB et al. Plate fixation and bone grafting of distal clavicle nonunions: radiologic and functional outcomes. Arch Orthop Trauma Surg 2016; 136: 15211529

[14] Gupta R, Sood M, Malhotra GD et al. Functional outcome of modified Weaver Dunn technique for acromioclavicular joint dislocation. Indian J Orthop 2018; 52: 418-422

[15] Seiler JG III, Jupiter J. Intercalary tri-cortical iliac crest bone grafts for the treatment of chronic clavicular nonunion with bony defect. J Orthop Tech 1993; 1: 19-22

[16] Simpson NS, Jupiter JB. Clavicular nonunion and malunion: evaluation and surgical management. J Am Acad Orthop Surg 1996; 4: 1-8

[17] Momberger NG, Smith J, Coleman DA. Vascularized fibular grafts for salvage reconstruction of clavicle nonunion. J Shoulder Elbow Surg 2000; 9: 389-394

[18] Chieh-Ting Huang T, Sabbagh MD, Lu CK et al. The vascularized medial femoral condyle free flap for reconstruction of segmental recalcitrant nonunion of the clavicle. J Shoulder Elbow Surg 2019; 28: 2364-2370

[19] Rollo G, Pichierri P, Marsilio A et al. The challenge of nonunion after osteosynthesis of the clavicle: is it a biomechanical or infection problem? Clin Cases Miner Bone Metab 2017; 14: 372378

[20] McKnight B, Heckmann N, Hill JR et al. Surgical management of midshaft clavicle nonunions is associated with a higher rate of short-term complications compared with acute fractures. J Shoulder Elbow Surg 2016; 25: 1412-1417

[21] McGahan JP, Rab GT, Dublin A. Fractures of the scapula. J Trauma 1980; 20: 880-883

[22] Lantry JM, Roberts CS, Giannoudis PV. Operative treatment of scapular fractures: a systematic review. Injury 2008; 39: 271283

[23] Tatro JM, Gilbertson JA, Schroder LK et al. Five to ten-year outcomes of operatively treated scapular fractures. J Bone Joint Surg Am 2018; 100: 871-878

[24] Wang WZ, Zhang HM, Han L. [Internal fixation for unstable scapular fracture]. Zhongguo Gu Shang 2012; 25: 687-689

[25] Lewis S, Argintar E, Jahn R et al. Intra-articular scapular fractures: outcomes after internal fixation. J Orthop 2013; 10: 188-192

[26] Zahid M, Rashid EH, Inam $\mathrm{H}$ et al. Outcomes of open reduction and internal fixation in displaced intra-articular scapular fractures: a case series. Acta Orthop Belg 2017; 83: 16-21 
[27] Nork SE, Barei DP, Gardner M] et al. Surgical exposure and fixation of displaced type IV, V and VI glenoid fractures. J Orthop Trauma 2008; 22: 487-493

[28] Court-Brown CM, Clement ND, Duckworth AD et al. The spectrum of fractures in the elderly. J Bone Joint Surg $\mathrm{Br} 2014$; 96: 366-372

[29] Krettek C, Wiebking U. Proximale Humerusfraktur. Ist die winkelstabile Plattenosteosynthese der konservativen Behandlung überlegen? Unfallchirurg 2011; 114: 1059-1067

[30] Bogner R, Ortmaier R, Moroder P et al. Minimally invasive treatment of displaced proximal humeral fractures in patients older than 70 years using the humerusblock. Biomech Res Int 2016; 2016: 6541849

[31] Plecko M, Kraus A. Internal fixation of proximal humerus fractures using the locking proximal humerus plate. Oper Orthop Traumatol 2005; 17: 25-50

[32] Brunner F, Sommer C, Bahrs C et al. Open reduction and internal fixation of proximal humerus fractures using a proximal humeral locked plate: a prospective multicenter analysis. J Orthop Trauma 2009; 23: 163-172

[33] Acklin YP, Stoffel K, Sommer C. A prospective analysis of the functional and radiological outcomes of minimal invasive plating in proximal humerus fractures. Injury 2013; 44: 456-460

[34] Rothstock S, Plecko M, Kloub M et al. Biomechanical evaluation of two intramedullary nailing techniques with different locking options in a three-part fracture proximal humerus model. Clin Biomech 2012; 27: 686-691

[35] Kloub M, Holub K, Urban J et al. Intramedullary nailing of displaced four-part fractures of the proximal humerus. Injury 2019; 50: 1978-1985

[36] Erdle B, Izadpanah K, Eberbach $\mathrm{H}$ et al. [Primary fracture prostheses and reverse shoulder arthroplasty in complex humeral head fractures: an alternative to joint-preserving osteosynthesis?] Orthopade 2018; 47: 410-419

[37] Shannon SE, Wagner ER, Houdek MT et al. Reverse shoulder arthroplasty for proximal humeral fractures: outcomes comparing primary reverse for fracture versus arthroplasty after failed osteosynthesis. J Shoulder Elbow Surg 2016; 25: 16551660
[38] Cicak N, Klobucar H, Medancic N. Reverse shoulder arthroplasty in acute fractures provides better results than in revision procedures for fracture sequelae. Int Orthop 2015; 39: 343348

[39] Santana F, Alentorn-Geli E, Girro P et al. Reverse shoulder arthroplasty for fracture sequelae: how the initial fracture treatment influences the outcomes of joint replacement. Acta Orthop Traumatol Turc 2019; 53: 278-281

[40] Jost B, Spross C, Grehn H et al. Locking plate fixation of fractures of the proximal humerus: analysis of complications, revision strategies and outcome. J Shoulder Elbow Surg 2013; 22: 542-549

[41] Hinds RM, Garner MR, Tran WH. Geriatric proximal humeral fracture patients show similar clinical outcomes to non-geriatric patients after osteosynthesis with endosteal fibular strut allograft augmentation. J Shoulder Elbow Surg 2015; 24: 889896

[42] Bosch U, Skutek M, Fremerey RW. Outcome after primary and secondary hemiarthroplasty in elderly patients with fracture of the proximal humerus. J Shoulder Elbow Surg 1998; 7 : 479-484

[43] Hussey MM, Hussey SE, Mighell MA. Reverse shoulder arthroplasty as a salvage procedure after failed internal fixation of fractures of the proximal humerus: outcomes and complications. Bone Joint J 2015; 97-B: 967-972

[44] Shannon SF, Wagner ER, Houdek MT et al. Reverse shoulder arthroplasty for proximal humeral fractures: outcomes comparing primary reverse arthroplasty for fracture versus reverse arthroplasty after failed osteosynthesis. J Shoulder Elbow Surg 2016; 25: 1655-1660

Bibliografie

DOI https://doi.org/10.1055/a-1044-3934 online publiziert 06.02.2020 | OP-JOURNAL 2020; 36: 89-100 (c) Georg Thieme Verlag KG Stuttgart · New York ISSN 0178-1715 